\title{
Identification of a novel MYO7A mutation in Usher syndrome type 1
}

\author{
Ling Cheng ${ }^{1,2, *}$, Hongsong $\mathrm{Yu}^{1,3, *}$, Yan Jiang ${ }^{1}$, Juan $\mathrm{He}^{1}$, Sisi $\mathrm{Pu}^{1}$, Xin $\mathbf{L i}^{1}$ and $\mathrm{Li}$ \\ Zhang ${ }^{1}$ \\ ${ }^{1}$ The First Affiliated Hospital of Chongqing Medical University, Chongqing Key Laboratory of Ophthalmology and Chongqing \\ Eye Institute, Chongqing, P. R. China \\ ${ }^{2}$ Department of Ophthalmology, Yongchuan Hospital, Chongqing Medical University, Chongqing, P. R. China \\ ${ }^{3}$ Department of Immunology, Special Key Laboratory of Gene Detection \& Therapy of Guizhou Province, Zunyi Medical \\ University, Guizhou, P. R. China \\ *These authors have contributed equally to this work \\ Correspondence to: Li Zhang, email: Zhangli298@hotmail.com
}

Keywords: Usher syndrome; USH 1 family; MYO7A; novel mutation

Received: June 21, $2017 \quad$ Accepted: December 05, $2017 \quad$ Published: December 19, 2017

Copyright: Cheng et al. This is an open-access article distributed under the terms of the Creative Commons Attribution License 3.0 (CC BY 3.0), which permits unrestricted use, distribution, and reproduction in any medium, provided the original author and source are credited.

\section{ABSTRACT}

Usher syndrome (USH) is an autosomal recessive disease characterized by deafness and retinitis pigmentosa. In view of the high phenotypic and genetic heterogeneity in USH, performing genetic screening with traditional methods is impractical. In the present study, we carried out targeted next-generation sequencing (NGS) to uncover the underlying gene in an USH family ( 2 USH patients and 15 unaffected relatives). One hundred and thirty-five genes associated with inherited retinal degeneration were selected for deep exome sequencing. Subsequently, variant analysis, Sanger validation and segregation tests were utilized to identify the disease-causing mutations in this family. All affected individuals had a classic USH type I (USH1) phenotype which included deafness, vestibular dysfunction and retinitis pigmentosa. Targeted NGS and Sanger sequencing validation suggested that USH1 patients carried an unreported splice site mutation, c.5168+1G>A, as a compound heterozygous mutation with c.6070C $>$ T (p.R2024X) in the MY07A gene. A functional study revealed decreased expression of the MYO7A gene in the individuals carrying heterozygous mutations. In conclusion, targeted next-generation sequencing provided a comprehensive and efficient diagnosis for USH1. This study revealed the genetic defects in the MYO7A gene and expanded the spectrum of clinical phenotypes associated with USH1 mutations.

\section{INTRODUCTION}

Usher syndrome (USH) is recognized as the most common form of hereditary deaf-blindness [1]. USH is an autosomal recessive disease that is characterized by clinical and genetic heterogeneity. The frequency of USH has been reported to range from 3.8 to $6.2 / 100$ 000 in different countries, such as Spain, Denmark and the United States [2-4]. The prevalence of USH in China has not been reported. USH can be subdivided into the 3 following clinical types: USH type I (USH1), USH type II (USH2), and USH type III (USH3). The classification of USH is mainly based on the severity and progression of hearing loss (HL), the status of vestibular function and age at onset of retinitis pigmentosa (RP). Patients with USH1 typically have profound and congenital HL, which is further accompanied by vestibular areflexia and progressive RP of prepubertal onset. Patients with USH2 present with moderate-to-severe HL with downsloping pure tone audiograms, normal vestibular function, 
and RP in adolescence. USH3 is very rare and variable in presentation. It may resemble USH1 or USH2, and vestibular dysfunction may be present [5].

For USH1, at least seven different loci can be mapped (USH1B-USH1H), but only the five following genes have been isolated: myosin VIIa (MYO7A), cadherin-23 (CDH23), protocadherin-15 (PCDH15), harmonin (USH1C), and SANS (USH1G) $[6,7]$. A previous study has reported that USH1B is the most common form of USHI and is responsible for approximately $70 \%$ of all cases [8]. Meanwhile, $M Y O 7 A$ is mainly responsible for USH1B and accounts for approximately $50 \%$ of all pathogenic mutations $[9,10]$, followed by four other mutated genes (CDH23, PCDH15, USH1C, and $U S H 1 G)$. The MYO7A gene is located on the long arm of chromosome 11 at position 13.5 (11q13.5). Human MYO7A comprises 49 exons spanning approximately 120 $\mathrm{kb}$ of genomic DNA. MYO7A codes for a 2215 amino acid protein, myosin VIIa, which has a molecular mass of 254 $\mathrm{kDa}$ [11]. Defects in $M Y O 7 A$ result in a wide phenotypic spectrum, including RP, sensorineural hearing impairment and vestibular dysfunction [12-14]. Most of these MYO7A mutations (more than 95\%) cause Usher syndrome type 1. Successful identification of USH1 causative mutation provides more probability for elucidating the underlying pathophysiology of USH.

Compared with traditional approaches including direct sequencing and linkage disequilibrium, nextgeneration sequencing (NGS) has been demonstrated as a significant improvement that provides precise diagnostic information and extends the possibility of targeted treatments. Recently, studies have confirmed that specific genes (TMC1, PCDH15, MYO7A and MYO15A) were associated with hereditary HL in families by the NGS method [15]. Furthermore, NGS techniques have also been applied to identify 100 retinal disease genes, resulting in a higher detection rate of approximately $50 \%$ for RP cases [16-18]. Additionally, NGS is much faster, more costefficient and more accurate than traditional methods [19, 20]. In this study, a mutation screening of 135 candidate genes associated with inherited retinal degeneration, including RP and USH, was carried out on a proband using targeted NGS. We identified a novel MYO7A splicing site mutation (c.5168+1G $>$ A) in a Chinese USH1 family, and found that the compound heterozygous mutations c. $6070 \mathrm{C}>\mathrm{T}$ (p.R2024X) and c. $5168+1 \mathrm{G}>\mathrm{A}$ in the $M Y O 7 A$ gene could lead to USH1.

\section{RESULTS}

\section{Clinical phenotype of the Chinese Usher syndrome pedigree}

Two affected members and fifteen unaffected individuals in an USH1 family were enrolled in this study. In this family, the proband had an affected brother, while the parents had no symptoms or signs of USH1 (Figure 1). The affected individuals included in the study suffered from vestibular dysfunction manifested by delayed walking (after two years old). All affected individuals showed profound bilateral sensorineural hearing impairment with vestibular response according

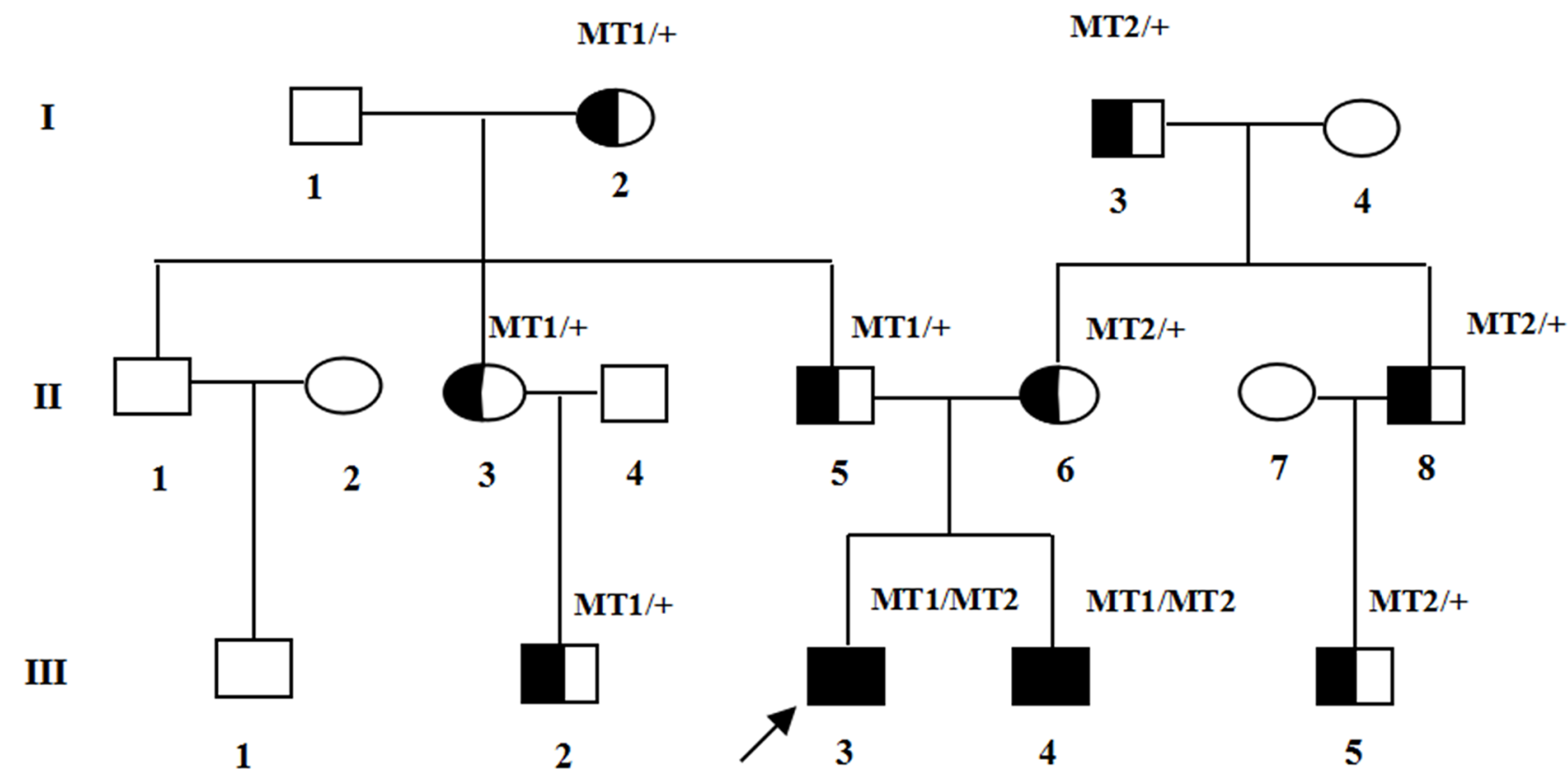

Figure 1: Pedigree of the family with USH1. Normal males and female are shown with empty squares and circle, respectively. Affected patient is shown with filled symbols. The proband (III:3) is indicated by arrow. MT: mutant type. MT1: c.5168+1G $>$ A; MT2: c. $6070 \mathrm{C}>\mathrm{T}$ (p.R2024X). 
to their pure tone audiometry results (Figure 2A). Next, detailed ophthalmological examinations were conducted. Fundus photography showed atrophy of the retinal pigment epithelium, attenuated arteries, pallor of the optic disc, bone-spicule pigmentation in the retinal periphery, and all typical signs related to RP (Figure 2B). The optical coherence tomography (OCT) presentations of proband III:3 and proband III:4 individuals exhibited cystoid macular edema, macular retinoschisis and retinal thinning (Figure 2C). Visual fields were restricted, ranging from $10^{\circ}$ to $20^{\circ}$ (Figure $2 \mathrm{D})$. The best correct visual acuity (BCVA) of the two affected individuals ranged from 0.4 to 0.8 . According to the typical clinical presentation and results from the audiometric test and ophthalmic examinations, we concluded that the proband and his older brother exhibited autosomal recessive USH1.

\section{Targeted exome sequencing identified candidate mutations}

Targeted exome sequencing was performed for the proband sample from the USH1 family. The average depth of the targeted regions was at least 100 -fold, with an overall coverage of the targeted regions equal to $98.0 \%$ for 10 or more reads. The Burrows-Wheeler Aligner (BWA) program was used to process raw data, and numerous non-synonymous variants were identified by using the genome analysis toolkit (GATK) program. The most likely pathogenic mutations were identified through the following approaches: 1) Functional variants (insertion/ deletion: in coding sequence and splicing region, SNP: nonsense, splice site and missense). 2) The allele frequency of variants residing around or under 0.01 in any of the abovementioned databases. 3) Pathogenicity predictions
$\mathbf{A}$

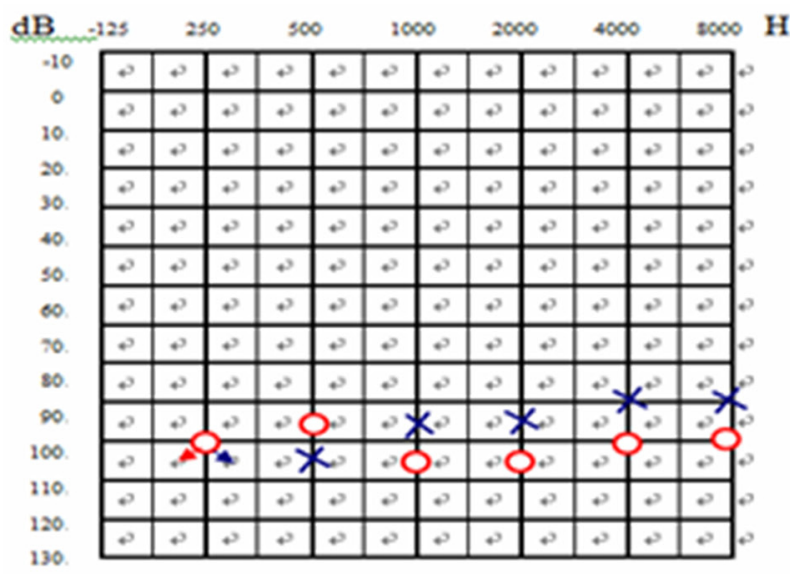

C

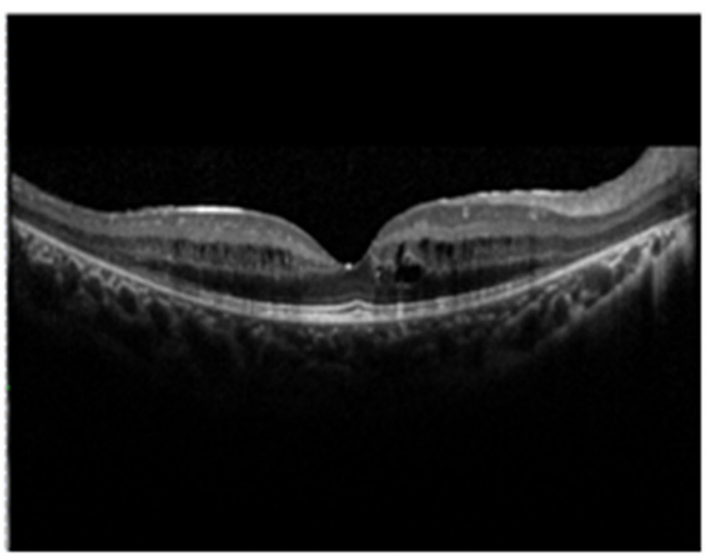

B

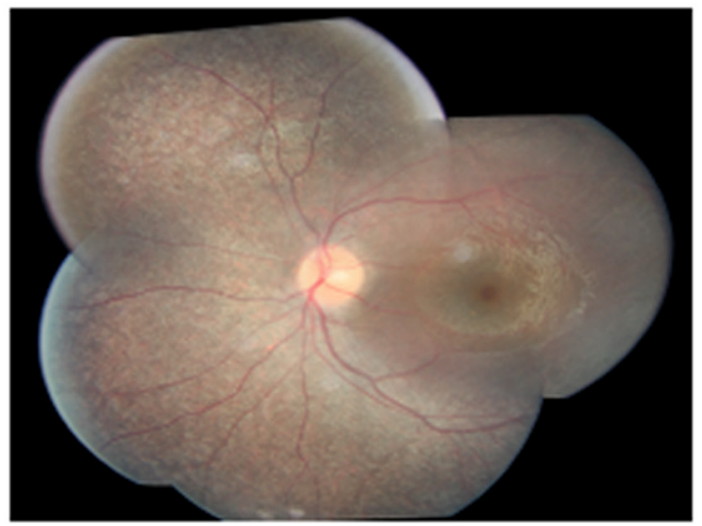

D

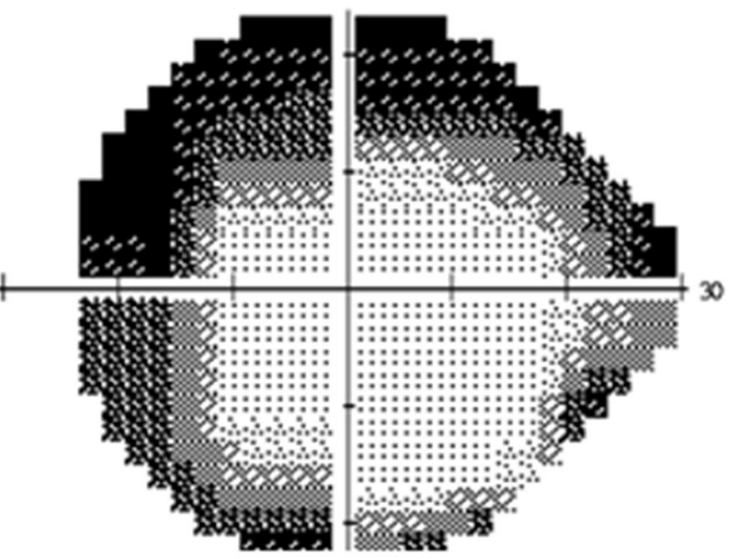

Figure 2: Pure tone audiogram, fundus photography, optical coherence tomography (OCT), and visual field of the proband. (A) Audiogram showed bilateral profound sensorineural hearing loss of proband III: 4(red, right ear; blue, left ear). (B) Fundus photography showed atrophy of the retinal pigment epithelium, attenuation of retinal arterioles, pallor of optic disc and bone-spicule pigmentation in the retinal periphery. (C) OCT revealed cystoid macular oedema, macular retinoschisis and thinned retina in proband. (D) Bilateral vision field got concentric narrowing, for the residual central visual field were 10-20 degrees. The extent of visual field ranged from $10^{\circ}$ to $20^{\circ}$. 
with three computational tools were conducted; if at least one of the programs predicted the variant to be possibly damaging, it was considered pathogenic. Finally, using the stepwise approach as described, only two compound heterozygous mutations in $M Y O 7 A$ were identified in this pedigree (c.6070C > T (p.R2024X) and c.5168+1G>A).

\section{Validation of the mutations by sanger sequencing confirmation}

The NGS results of the sample from the proband (III:2) showed probable defects in the MYO7A gene. We performed Sanger sequencing to validate the two heterozygous variants in $M Y O 7 A$ that were identified in the proband. Meanwhile, Sanger sequencing was also used to screen the same mutation sites in unaffected family members and the affected sibling. We confirmed two compound heterozygous mutations in this USH1 family, and one mutation in MYO7A has not been reported in any of the four SNP databases (dbSNP (Build 138), HapMap database, 1000 Genome Project and a local control database).

The proband and the affected sibling harbored compound heterozygous $M Y O 7 A$ mutations c.6070C $>\mathrm{T}$ (p.R2024X) and c.5168+1G $>$ A from the parents. c. $5168+1 \mathrm{G}>\mathrm{A}$, a heterozygous splice site mutation (Figure 3), was found in exon 37 of $M Y O 7 A$ in the proband (III:3) and also in his affected sibling (III:4), unaffected father (II:5), aunt (II:3), cousin (III:2) and unaffected grandmother (I:2). Another mutation, c.6070C $>$ T (p.R2024X), a nonsense mutation (Figure 3), was identified in exon 45 of MYO7A in the proband (III:3) and also in his affected sibling (III:4), his mother (II:6), uncle (II:8), cousin (III:5) and his maternal grandfather (I:3). Mutation (c.5168+1G>A), confirmed by Sanger sequencing, was inherited from the grandmother (I:2), and (c.6070C > T (p.R2024X)) was inherited from the maternal grandfather (I:3).

\section{Analysis of MYO7A mutations}

Mutation c.6070C >T (p.R2024X), a substitution of $\mathrm{C}$ to $\mathrm{T}$ in exon 45 of the MYO7A gene has been reported in an earlier study [21]. This substitution is a nonsense variant predicted to alter an arginine into a stop codon at amino acid position 2024 of the myosin VIIa protein (total length: 2215 amino acids). Mutation p.R2024X might lead to dysfunction or reduction of myosin VIIa through truncation of the generated protein or nonsense-mediated mRNA decay (NMD), respectively.
Proband

$\mathbf{T}$

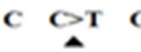

A G G C

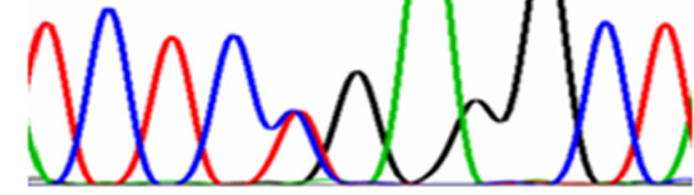

$\mathbf{T}$

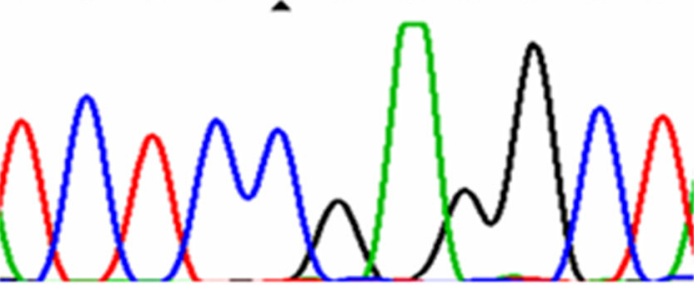

Father

Father

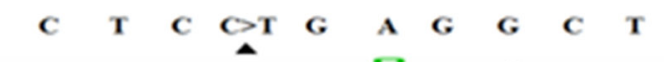

Mother

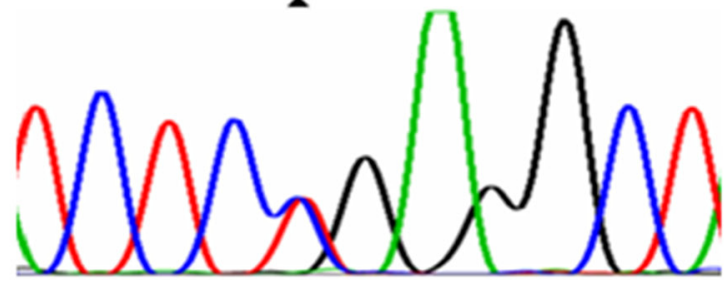

c. $6070 \mathrm{C}>\mathrm{T}(\mathrm{p} . \mathrm{R} 2024 \mathrm{X})$
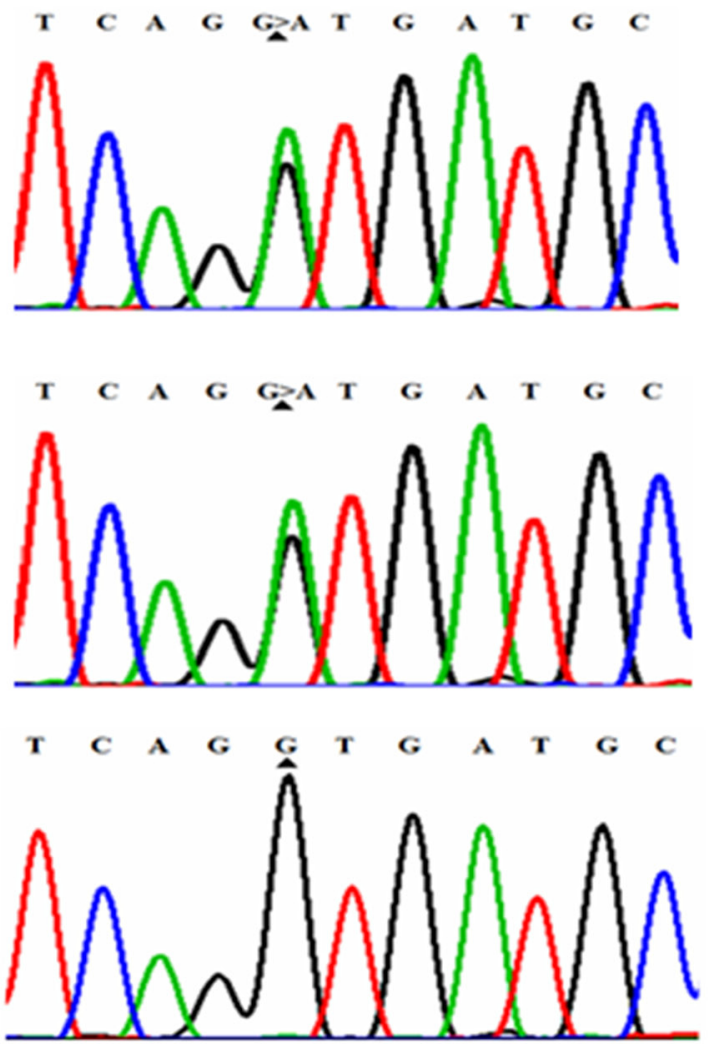

c. $5168+1 \mathrm{G}>\mathrm{A}$

Figure 3: Mutational analysis of MYO7A in USHI family. DNA sequencing profile showing the c.6070C $>$ T (p.R2024X) and c. $5168+1 \mathrm{G}>$ A mutations in MYO7A. Both variants co-segregated with the clinical phenotype. 
c. $5168+1 \mathrm{G}>\mathrm{A}$, a splicing site mutation, has not been reported in earlier studies or in any other SNP database. Mutation c. $5168+1 \mathrm{G}>\mathrm{A}$ was a change from $\mathrm{G}$ to $\mathrm{A}$ at the conserved donor splice site in exon 37. According to online splice site prediction software (splice), mutation c. $5168+1 \mathrm{G}>\mathrm{A}$ was predicted to abolish the splice donor site, resulting in a premature termination codon (PTC) located at c. $5168+32$ to c. $5168+34$ in exon 37 , TAG, leading to NMD and/or the generation of a truncated protein. The two compound heterozygous mutations c.6070C $>$ T (p.R2024X) and c.5168+1G $>$ A in $M Y O 7 A$ were highly conserved (GERP++ scores of 3.70 and 3.92, respectively). In summary, we successfully identified two truncating variants that were predicted to introduce a premature stop codon and therefore predicted to disrupt gene and protein function. The two mutations detected with NGS were verified by Sanger sequencing. Familial segregation analyses were also performed by polymerase chain reaction (PCR) and Sanger sequencing (Figure 1).

\section{Analysis of mutation pathogenicity in gene MYO7A}

The aforementioned result showed two compound heterozygous mutations in this USH1 family. Mutation c.6070C $>$ T (p.R2024X), reported in an earlier study, is a nonsense mutation, whereas mutation c.5168+1G $>$ A is a novel mutation, which could potentially affect a consensus donor splice site essential for splicing of exon 37. To assess the possible effect of the mutations on the expression of $M Y O 7 A$, RT-PCR was performed to analyze the total lymphocyte RNA obtained from the affected sibling and the parents. The results showed that premature stop codons, which were predicted to create two mutations, apparently activate the NMD response, resulting in decreased MYO7A mRNA expression (Figure 4, $\mathrm{P}<0.05$ ). The two predicted compound heterozygous mutations can cause complete dysfunction of myosin VIIa in the same subject and lead to the observed phenotype in both the proband and affected sibling.

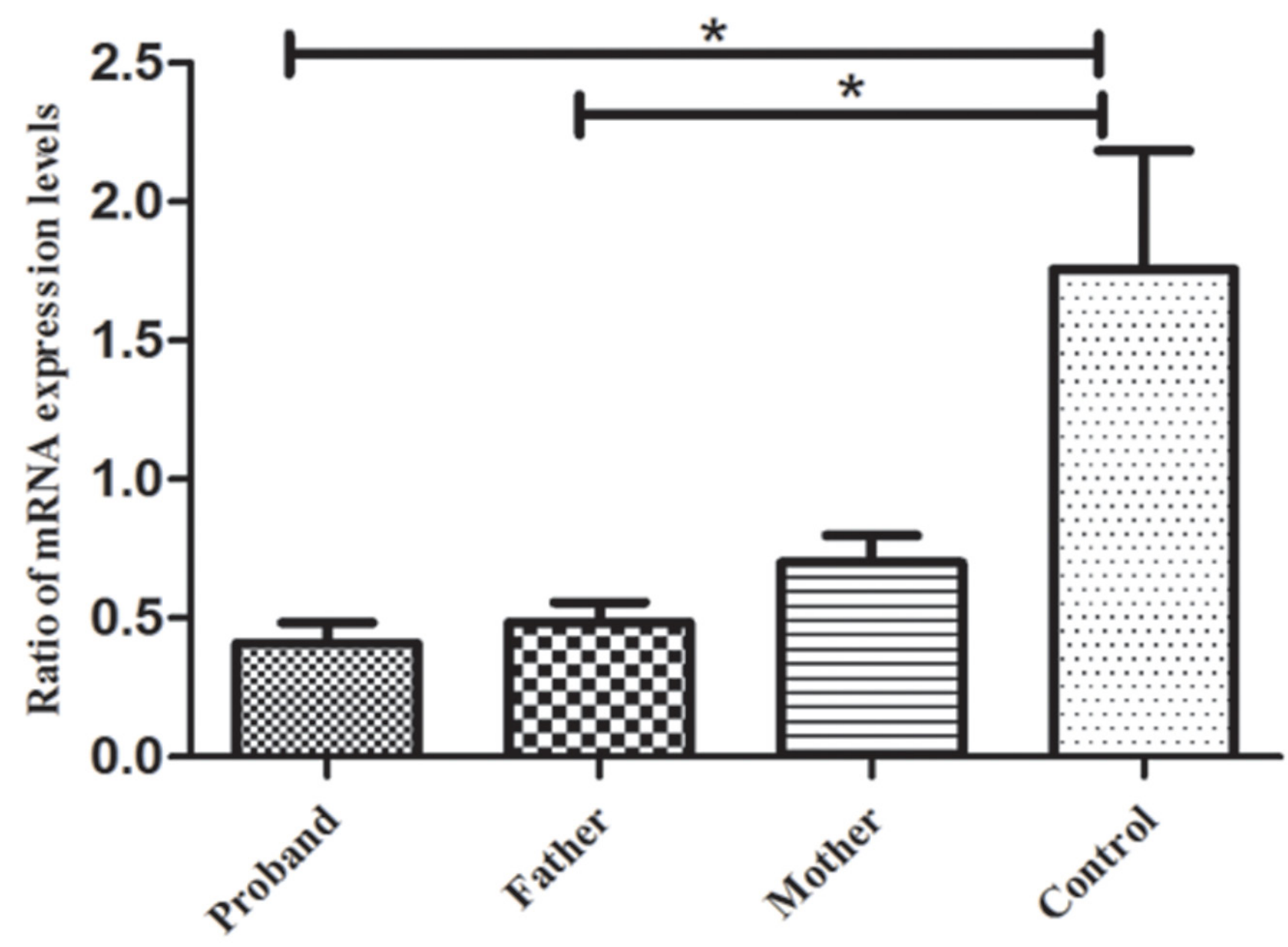

Figure 4: RT-PCR results for gene MYO7A. The results indicated that the mRNA expression levels of gene MYO7A in the proband and her father were significantly lower than those of the normal controls $(p<0.05)$, but no significant difference was found between the proband's mother and the normal controls $(p>0.05)$. ${ }^{*} p<0.05$, statistical significance was determined with a Student's $t$ test. 


\section{DISCUSSION}

In this Chinese USH1 family, we identified one reported mutation (c.6070C $>\mathrm{T}$ (p.R2024X)) and one novel splice mutation (c.5168+1G $>$ A) using NGS methods. Both the proband and his sibling carried compound heterozygous mutations in MYO7A (c.5168+1G $>$ A and c.6070C $>$ T (p.R2024X)). These mutations were considered pathogenic mainly due to the following reasons: 1) mutation co-segregation in this family; 2) pathogenicity predictions with three computational tools (Mutation Taster, PolyPhen-2 and Sorting Intolerant From Tolerant (SIFT); and 3) the mutations were not seen in the 1000-Genomes Project or any other single nucleotide polymorphism database.

The protein myosin VIIa contains three typical domains (Figure 5): an N-terminal head domain (1729) or motor, a neck region containing five isoleucine glutamine motifs (IQ) (IQ1-IQ5: 745-857), and a tail beginning with a short coiled-coil region domain for dimerization (858-935), followed by two MyTH4-FERM repeat elements (1017-1602) (1747-2205), separated by an SH3 domain (1603-1672) [22]. Myosin VIIa plays an important role in the retinal pigment epithelium and photoreceptor cells, as well as in the inner ear, as a type of molecular motor. In photoreceptor cells, myosin VIIa displays an active function in the transport of opsin from the inner segment to the outer segment of photoreceptors through the connecting cilium [23]. In the human retina, myosin VIIa functions actively in the migration of RPE melanosomes, phagocytosis of photoreceptor cell outer segment tips and opsin transport through the cilium of these photoreceptors $[24,25]$. The nonsense mutation c. $6070 \mathrm{C}>\mathrm{T}$ (p.R2024X) and the splice site mutation c. $5168+1 \mathrm{G}>\mathrm{A}$ may cause complete functional loss of myosin VIIa protein. The nonsense mutation, c. $6070 \mathrm{C}>\mathrm{T}$ (p.R2024X), can generate a PTC, containing only 2024 amino acids with truncation of the last 191 amino acids (Figure 5). This mutant protein lacks the tail region. The tail domain of MYO7A is believed to be largely responsible for its function [26]. Mutation c.5168+1G $>A$ is predicted to lead to a PTC (TAG, located at c. $5168+32$ to c.5168+34 in exon 37) after a $G$ to A transition at the splice acceptor site. As a result, mutation c. $5168+1 \mathrm{G}>\mathrm{A}$ may be involved in abnormal RNA processing and trigger a truncated MYO7A protein. Both identified compound heterozygous mutations could lead to a truncated protein that disrupts the normal function of myosin VIIa, which can lead to the observed phenotype of the proband and the affected sibling.

For hereditary diseases including USH1, comprehensive molecular testing can help to elucidate the molecular pathogenesis, provide early diagnosis, and explain the severe clinically observed symptoms. Using targeted NGS, we can fulfill ethnicity-specific diagnosis of USH. However, our study has several limitations. First, NGS is not a routine application that can be used in clinic because of the cost, infrastructural logistics, strategies for patient selection, and interpretation of genetic datasets [27]. In addition, compared with whole genome sequencing, our approach detected only 135 genes related to inherited retinal degeneration. NGS technology cannot identify mutations in deep intronic regions, structural variations or large copynumber. Furthermore, members in this family with both mutations had severe symptoms and signs of USH1, while others with one or without mutations were completely normal. These phenotypic differences might be partially due to the complex interactions between the two mutations. Therefore, we should perform additional functional studies to determine the complex relationship between the two mutations and the pathogenesis of distinct mutations.

In conclusion, we determined that the compound heterozygous mutations, c.6070C $>\mathrm{T} \quad$ (p.R2024X) and c. $5168+1 \mathrm{G}>\mathrm{A}$, could result in USH1 in a Chinese family. Our finding expands the spectrum of clinical phenotypes of mutations, which can help us better understand the genotype-phenotype relationship in this disease, and provides the potential for early molecular diagnoses, accurate genetic counseling, and optimal rehabilitation.

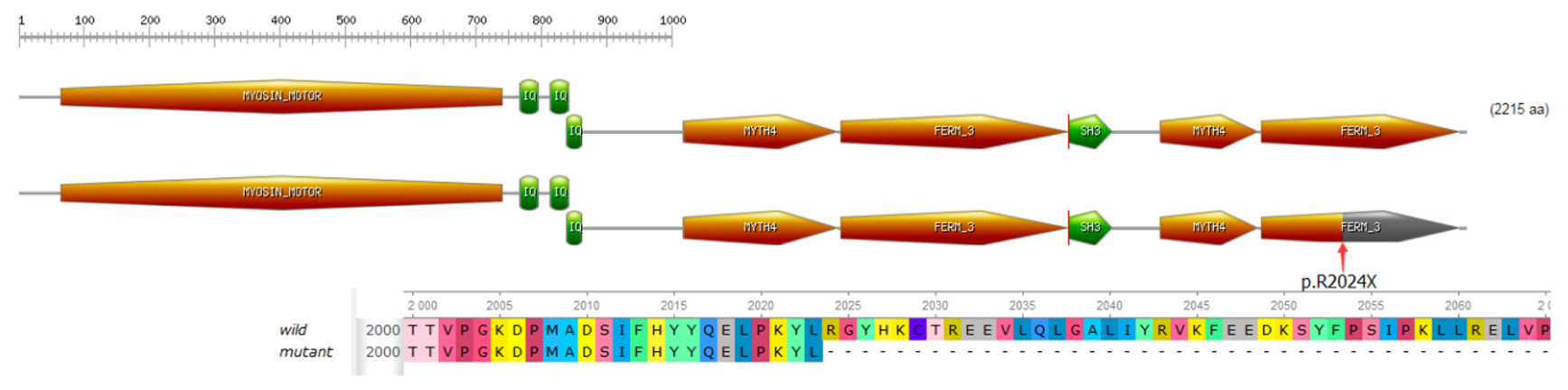

Figure 5: Domain structure of myosin VIIa. The nonsense mutation introduces a premature stop codon which is predicted to truncate the protein within the $\mathrm{N}$-terminal motor domain. 


\section{MATERIALS AND METHODS}

\section{Subjects}

A three-generation consanguineous Chinese family diagnosed with USH1 was enrolled in this study. The pedigree of the family contains 17 members and is shown in Figure 1. A full medical and family history was recorded for all family members, mainly including intelligence and growth development, hearing impairment and visual loss. A routine ophthalmologic examination, including BCVA, intraocular pressure, slit-lamp biomicroscopy, and dilated indirect ophthalmoscopy, was performed in the department of ophthalmology at the First Affiliated Hospital of Chongqing Medical University (Chongqing, China). The proband and affected patient underwent an additional eye examination, including visual field, fundus photography, OCT, visual evoked potential, synoptophore and visual function examination. An otology examination was also performed, including audiometric examinations and vestibular evaluation by otolaryngologists. All participating individuals provided written informed consent before donating venous blood. This study was approved by the Local Ethics Research Council and was conducted according to the principles of the Declaration of Helsinki. All experiments were conducted in accordance with the approved guidelines and regulations.

\section{Targeted region capture and NGS}

A total of 135 inherited retinal degeneration-related genes (Supplementary Table 1) were selected using a solution-based sequence capture custom enrichment kit (GenCap, MyGenostics, Beijing, China). Peripheral blood samples were collected from the proband, and genomic DNA extraction was performed using a QIAamp DNA Blood Mini Kit (Qiagen, Valencia, CA, USA). The quantity and quality of the DNA were verified using a NanoDrop 2000 (Thermo Fisher Scientific, Delaware, USA). Next, genomic DNA $(1 \mu \mathrm{g})$ was fragmented into $150-300 \mathrm{bp}$ by a Covaris sonicator (Covaris S2, Massachusetts, USA). After end-repair and adapter ligation, the fragments underwent 4 cycles of PCR amplification with Illumina PCR primers. The purified PCR products $(500 \mathrm{ng}$ ) were hybridized to the GenCapTM probe (in solution) for 16 hours. After hybridization, washing, and elution, the eluted fraction underwent 14 cycles of PCR amplification and was subsequently subjected to Qubit and quantitative PCR to estimate the magnitude of enrichment. Each eluted, enriched DNA sample was then sequenced on HiSeq2000 Analyzers (Illumina, San Diego, USA) for 90-bp pairedread sequencing, providing an average coverage depth of each sample of at least 100-fold. Image analysis and base calling was performed using Illumina Pipeline (version 1.3.4) with default parameters.

\section{Bioinformatics analysis}

Bioinformatics analysis was conducted as previously described [28]. To identify the underlying variants of the proband, the 90-bp clean reads were subjected to alignment with the reference human genome (UCSC hg19) database (http://hgdownload.cse.ucsc.edu/ goldenPath/hg19/bigZips/) using the BWA Multi-Vision software package (http://bio-bwa. sourceforge.net/) [29]. Next, single and multiple nucleotide variants, including small insertion or deletions, were identified by GATK software [30]. Several common variant databases, such as the 1000 Genomes database (http://www.1000genomes. org/), dbSNP (Build 138) (NCBI, http://www.ncbi.nlm. nih.gov/SNP/ [in the public domain]), HapMap database (ftp://ftp.ncbi.nlm.nih.gov/hapmap), and a local control database, were used to remove all variants with an allele frequency above $1 \%$.

\section{Mutation validation and silico analyses}

After filtration, variants were subsequently verified by PCR and Sanger sequencing of samples from the proband and family members. Peripheral blood samples from the family members were collected in ethylenediaminetetraacetic acid (EDTA) anti-coagulated tubes and stored at $-80^{\circ} \mathrm{C}$ until used. Genomic DNA was extracted by a QIAamp DNA Blood Mini Kit (Qiagen, Valencia, CA, USA) according to the manufacturer's instructions. Sanger sequencing was applied to perform segregation tests for all participants. Amplification of the target DNA sequence in the MYO7A gene was analyzed by PCR using the primers presented in Supplementary Table 2. Three online software programs, Polymorphism Phenotyping (PolyPhen-2, http://genetics.bwh.harvard. edu/pph2/), SIFT (http://sift.bii.a-star.edu.sg/), and Mutation Taster (http://www.mutationtaster.org), were used to evaluate the possible pathogenic effects of the mutations.

\section{Real-time PCR}

Total RNA was isolated from peripheral blood mononuclear cells (PBMC), which were obtained from the USH1 family members, with TRIzol (Invitrogen, San Diego, California, USA). Reverse transcription was performed by a transcriptase kit (Takara, Dalian, China). Relative mRNA expression was measured by a 7500 Real-Time PCR Instrument (ABI). MYO7A and $\beta$-actin expression levels were tested by the following primers ( $M Y O 7 A$, forward primer: 5'-CGCTACCGGGACCACCTC-3' and reverse primer: 5'-GGGCATCTCCCCTATCTTC-3'; $\beta$-actin, forward primer: 5'-GGATGCAGAAGGAGATCACTG-3' and reverse primer: 5'-CGATCCACACGGAGTACTT-3'). The relative expression level of MYO7A was computed using the $2^{-\Delta \Delta C t}$ method. 


\section{Author contributions}

L.Z., L.C. and H.Y. designed experiment; L.C., Y.J. and H.Y. performed laboratory work and analyzed data; L.C., H.Y. and L.Z. wrote the manuscript; L.C. and H.Y. prepared figures; J.H., S.P. and X.L. collected blood of all patients and controls. All authors read and approved the final manuscript.

\section{ACKNOWLEDGMENTS} study.

We are grateful to all donors enrolled in the present

\section{CONFLICTS OF INTEREST}

The authors declare no competing interest exist.

\section{FUNDING}

This work was supported by the National Natural Science Foundation Project (81670844, 81200678), the Fundamental and Advanced Research Program of Chongqing (cstc2015jcyjA10022, csts2016jcyjA0546), the Science and Technology Project of Chongqing Municipal Education Commission (KJ1500236, KJ120304), the Scientific Research Program of the Science and Technology Commission of Yuzhong District of Chongqing (20150102), the Excellent Talent Support Program of Guizhou Provincial Education Department (QJH-KY-2017-077) and the National Key Clinical Specialties Construction Program of China.

\section{REFERENCES}

1. Smith RJ, Berlin CI, Hejtmancik JF, Keats BJ, Kimberling WJ, Lewis RA, Moller CG, Pelias MZ, Tranebjaerg L. Clinical diagnosis of the Usher syndromes. Usher syndrome consortium. Am J Med Genet. 1994; 50:32-8. https://doi. org/10.1002/ajmg.1320500107.

2. Espinos C, Najera C, Millan JM, Ayuso C, Baiget M, PerezGarrigues H, Rodrigo O, Vilela C, Beneyto M. Linkage analysis in Usher syndrome type I (USH1) families from Spain. J Med Genet. 1998; 35:391-8.

3. Rosenberg T, Haim M, Hauch AM, Parving A. The prevalence of Usher syndrome and other retinal dystrophyhearing impairment associations. Clin Genet. 1997; 51:314-21.

4. Hope CI, Bundey S, Proops D, Fielder AR. Usher syndrome in the city of Birmingham--prevalence and clinical classification. Br J Ophthalmol. 1997; 81:46-53.

5. Besnard T, Garcia-Garcia G, Baux D, Vache C, Faugere V, Larrieu L, Leonard S, Millan JM, Malcolm S, Claustres M, Roux AF. Experience of targeted Usher exome sequencing as a clinical test. Mol Genet Genomic Med. 2014; 2:30-43. https://doi.org/10.1002/mgg3.25.

6. Ahmed ZM, Riazuddin S, Riazuddin S, Wilcox ER. The molecular genetics of Usher syndrome. Clin Genet. 2003; 63:431-44.

7. Petit C, Levilliers J, Hardelin JP. Molecular genetics of hearing loss. Annu Rev Genet. 2001; 35:589-646. https:// doi.org/10.1146/annurev.genet.35.102401.091224.

8. Adato A, Weil D, Kalinski H, Pel-Or Y, Ayadi H, Petit C, Korostishevsky M, Bonne-Tamir B. Mutation profile of all 49 exons of the human myosin VIIA gene, and haplotype analysis, in Usher 1B families from diverse origins. Am J Hum Genet. 1997; 61:813-21. https://doi. org/10.1086/514899.

9. Le Quesne Stabej P, Saihan Z, Rangesh N, Steele-Stallard HB, Ambrose J, Coffey A, Emmerson J, Haralambous E, Hughes Y, Steel KP, Luxon LM, Webster AR, BitnerGlindzicz M. Comprehensive sequence analysis of nine Usher syndrome genes in the UK national collaborative Usher study. J Med Genet. 2012; 49:27-36. https://doi. org/10.1136/jmedgenet-2011-100468.

10. Jaijo T, Aller E, Beneyto M, Najera C, Graziano C, Turchetti D, Seri M, Ayuso C, Baiget M, Moreno F, Morera C, Perez-Garrigues H, Millan JM. MYO7A mutation screening in Usher syndrome type I patients from diverse origins. J Med Genet. 2007; 44:e71. https://doi.org/10.1136/ jmg.2006.045377.

11. Kelley PM, Weston MD, Chen ZY, Orten DJ, Hasson T, Overbeck LD, Pinnt J, Talmadge CB, Ing P, Mooseker MS, Corey D, Sumegi J, Kimberling WJ. The genomic structure of the gene defective in Usher syndrome type Ib (MYO7A). Genomics. 1997; 40:73-9. https://doi.org/10.1006/ geno.1996.4545.

12. Liu XZ, Walsh J, Mburu P, Kendrick-Jones J, Cope MJ, Steel KP, Brown SD. Mutations in the myosin VIIA gene cause non-syndromic recessive deafness. Nat Genet. 1997; 16:188-90. https://doi.org/10.1038/ng0697-188.

13. Liu XZ, Walsh J, Tamagawa Y, Kitamura K, Nishizawa M, Steel KP, Brown SD. Autosomal dominant non-syndromic deafness caused by a mutation in the myosin VIIA gene. Nat Genet. 1997; 17:268-9. https://doi.org/10.1038/ng1197-268.

14. Weil D, Blanchard S, Kaplan J, Guilford P, Gibson F, Walsh J, Mburu P, Varela A, Levilliers J, Weston MD, Kelley PM, Kimberling WJ, Wagenaar M, et al. Defective myosin VIIA gene responsible for Usher syndrome type 1B. Nature. 1995; 374:60-1. https://doi.org/10.1038/374060a0.

15. Chen Y, Wang Z, Wang Z, Chen D, Chai Y, Pang X, Sun L, Wang $X$, Yang T, Wu H. Targeted next-generation sequencing in Uyghur families with non-syndromic sensorineural hearing loss. PLoS One. 2015; 10:e0127879. https://doi.org/10.1371/journal.pone.0127879.

16. Neveling K, Collin RW, Gilissen C, van Huet RA, Visser L, Kwint MP, Gijsen SJ, Zonneveld MN, Wieskamp N, de Ligt J, Siemiatkowska AM, Hoefsloot LH, Buckley MF, et 
al. Next-generation genetic testing for retinitis pigmentosa. Hum Mutat. 2012; 33:963-72. https://doi.org/10.1002/ humu. 22045.

17. O'Sullivan J, Mullaney BG, Bhaskar SS, Dickerson JE, Hall G, O'Grady A, Webster A, Ramsden SC, Black GC. A paradigm shift in the delivery of services for diagnosis of inherited retinal disease. J Med Genet. 2012; 49:322-6. https://doi.org/10.1136/jmedgenet-2012-100847.

18. Shanks ME, Downes SM, Copley RR, Lise S, Broxholme J, Hudspith KA, Kwasniewska A, Davies WI, Hankins MW, Packham ER, Clouston P, Seller A, Wilkie AO, et al. Nextgeneration sequencing (NGS) as a diagnostic tool for retinal degeneration reveals a much higher detection rate in earlyonset disease. Eur J Hum Genet. 2013; 21:274-80. https:// doi.org/10.1038/ejhg.2012.172.

19. Metzker ML. Sequencing technologies - the next generation. Nat Rev Genet. 2010; 11:31-46. https://doi. org/10.1038/nrg2626.

20. Corton M, Nishiguchi KM, Avila-Fernandez A, Nikopoulos K, Riveiro-Alvarez R, Tatu SD, Ayuso C, Rivolta C. Exome sequencing of index patients with retinal dystrophies as a tool for molecular diagnosis. PLoS One. 2013; 8:e65574. https://doi.org/10.1371/journal.pone.0065574.

21. Jacobson SG, Aleman TS, Sumaroka A, Cideciyan AV, Roman AJ, Windsor EA, Schwartz SB, Rehm HL, Kimberling WJ. Disease boundaries in the retina of patients with Usher syndrome caused by MYO7A gene mutations. Invest Ophthalmol Vis Sci. 2009; 50:1886-94. https://doi. org/10.1167/iovs.08-3122.

22. Jaijo T, Aller E, Oltra S, Beneyto M, Najera C, Ayuso C, Baiget M, Carballo M, Antinolo G, Valverde D, Moreno F, Vilela C, Perez-Garrigues $\mathrm{H}$, et al. Mutation profile of the MYO7A gene in Spanish patients with Usher syndrome type I. Hum Mutat. 2006; 27:290-1. https://doi.org/10.1002/humu.9404.
23. Liu X, Udovichenko IP, Brown SD, Steel KP, Williams DS. Myosin VIIa participates in opsin transport through the photoreceptor cilium. J Neurosci. 1999; 19:6267-74.

24. Udovichenko IP, Gibbs D, Williams DS. Actin-based motor properties of native myosin VIIa. J Cell Sci. 2002; 115:445-50.

25. El-Amraoui A, Petit C. Usher I syndrome: unravelling the mechanisms that underlie the cohesion of the growing hair bundle in inner ear sensory cells. J Cell Sci. 2005; 118:4593-603. https://doi.org/10.1242/jcs.02636.

26. Oliver TN, Berg JS, Cheney RE. Tails of unconventional myosins. Cell Mol Life Sci. 1999; 56:243-57.

27. Davies WI. Challenges using diagnostic next-generation sequencing in the clinical environment for inherited retinal disorders. Pers Med. 2014; 11:99-111.

28. Chen X, Zhao K, Sheng X, Li Y, Gao X, Zhang X, Kang X, Pan X, Liu Y, Jiang C, Shi H, Chen X, Rong W, et al. Targeted sequencing of 179 genes associated with hereditary retinal dystrophies and 10 candidate genes identifies novel and known mutations in patients with various retinal diseases. Invest Ophthalmol Vis Sci. 2013; 54:2186-97. https://doi.org/10.1167/iovs.12-10967.

29. Li H, Durbin R. Fast and accurate long-read alignment with Burrows-Wheeler transform. Bioinformatics. 2010; 26:58995. https://doi.org/10.1093/bioinformatics/btp698.

30. DePristo MA, Banks E, Poplin R, Garimella KV, Maguire JR, Hartl C, Philippakis AA, del Angel G, Rivas MA, Hanna M, McKenna A, Fennell TJ, Kernytsky AM, et al. A framework for variation discovery and genotyping using next-generation DNA sequencing data. Nat Genet. 2011; 43:491-8. https://doi. org/10.1038/ng.806. 